TRANSACTIONS OF THE

AMERICAN MATHEMATICAL SOCIETY

Volume 359, Number 6, June 2007, Pages 2577-2595

S 0002-9947(07)03967-0

Article electronically published on January 4, 2007

\title{
THE EULER CHARACTERISTIC OF THE WHITEHEAD AUTOMORPHISM GROUP OF A FREE PRODUCT
}

\author{
CRAIG JENSEN, JON MCCAMMOND, AND JOHN MEIER
}

\begin{abstract}
A combinatorial summation identity over the lattice of labelled hypertrees is established that allows one to gain concrete information on the Euler characteristics of various automorphism groups of free products of groups. In particular, we establish formulae for the Euler characteristics of: the group of Whitehead automorphisms $\mathrm{Wh}\left(*_{i=1}^{n} G_{i}\right)$ when the $G_{i}$ are of finite homological type; $\operatorname{Aut}\left(*_{i=1}^{n} G_{i}\right)$ and $\operatorname{Out}\left(*_{i=1}^{n} G_{i}\right)$ when the $G_{i}$ are finite; and the palindromic automorphism groups of finite rank free groups.
\end{abstract}

\section{INTRODUCTION}

Let $G=G_{1} * \cdots * G_{n}$, where the $G_{i}$ are non-trivial groups. There are various subgroups of $\operatorname{Aut}(G)$ and $\operatorname{Out}(G)$ (such as the Whitehead automorphism group) that are only defined with respect to a specified free product decomposition of $G$. In this article we calculate the Euler characteristics of several such groups. Postponing definitions for the moment, our main result is the following.

Theorem A. If $G=G_{1} * \cdots * G_{n}$ is a free product of groups where $\chi(G)$ is defined, then the Euler characteristic of the group of outer Whitehead automorphisms is

$$
\chi(\mathrm{OWH}(G))=\chi(G)^{n-2}=\left[\chi\left(G_{1}\right)+\cdots+\chi\left(G_{n}\right)-(n-1)\right]^{n-2} .
$$

As a consequence, the Euler characteristic of the group of Whitehead automorphisms is $\chi(\mathrm{WH}(G))=\chi(G)^{n-1}$.

A natural situation to consider is when each group $G_{i}$ is finite. In this case the vcd of $\operatorname{Aut}(G)$ is $n-1$ (independently established in [KV93] and MM96]) and $\mathrm{WH}(G)$ is a finite index subgroup of $\operatorname{AUT}(G)$. Theorem $\mathrm{A}$ thus implies:

Theorem B. If $G=G_{1} * \cdots * G_{n}$ is a free product of finite groups and $\Omega \subset \Sigma_{n}$ is the subgroup of the symmetric group given by permuting isomorphic groups $G_{i}$, then the Euler characteristics of the Fouxe-Rabinovitch automorphism group of $G$,

Received by the editors September 15, 2004 and, in revised form, February 9, 2005.

2000 Mathematics Subject Classification. Primary 20J06, 57M07.

The first author was partially supported by Louisiana Board of Regents RCS contract no. LEQSF-RD-A-39.

The second author was partially supported by NSF grant no. DMS-0101506.

The third author was partially supported by an AMS Centennial Research Fellowship. 
the full automorphism group of $G$ and the outer automorphism group of $G$ are:

$$
\begin{aligned}
\chi(\operatorname{FR}(G)) & =\chi(G)^{n-1} \prod_{i=1}^{n}\left|\operatorname{Inn}\left(G_{i}\right)\right|, \\
\chi(\operatorname{Aut}(G)) & =\chi(G)^{n-1}|\Omega|^{-1} \prod_{i=1}^{n}\left|\operatorname{Out}\left(G_{i}\right)\right|^{-1}, \\
\chi(\operatorname{Out}(G)) & =\chi(G)^{n-2}|\Omega|^{-1} \prod_{i=1}^{n}\left|\operatorname{Out}\left(G_{i}\right)\right|^{-1} .
\end{aligned}
$$

The automorphism groups mentioned in these theorems are defined as follows.

Definition 1.1 (Groups of automorphisms). Let $G_{1} * \cdots * G_{n}$ be a free product of groups. As usual, we use $\operatorname{Aut}(G)$ to denote the full automorphism group of $G$ and $\operatorname{OuT}(G)$ for the group of outer automorphisms. If $g_{i} \in G_{i} \backslash\{1\}$ let $\alpha_{j}^{g_{i}}$ denote the automorphism described by

$$
\alpha_{j}^{g_{i}}(g)=\left\{\begin{array}{cl}
g, & g \in G_{k} \text { where } k \neq j \\
g^{g_{i}}, & g \in G_{k} \text { where } k=j .
\end{array}\right.
$$

The Fouxe-Rabinovitch group $\operatorname{FR}(G)$ is the subgroup of $\operatorname{AUT}(G)$ generated by $\left\{\alpha_{j}^{g_{i}} \mid i \neq j\right\}$. The group of Whitehead automorphisms is generated by all the $\alpha_{j}^{g_{i}}$ (including the possibility that $i=j$ ). In general, the symmetric automorphisms of a free product consist of those automorphisms that send each factor group to a conjugate of a (possibly different) factor. It is well-known that the group $\mathrm{WH}(G)$ is the kernel of the map $f: \Sigma \operatorname{Aut}(G) \rightarrow \operatorname{OUT}\left(\prod_{i} G_{i}\right)$ and $\operatorname{FR}(G)$ is the kernel of the map $g: \Sigma \operatorname{Aut}(G) \rightarrow \operatorname{AUT}\left(\prod_{i} G_{i}\right)$, where $\Sigma \operatorname{Aut}(G)$ denotes the group of symmetric automorphisms.

A quick chasing of definitions shows that the images of $\mathrm{FR}(G)$ and $\mathrm{WH}(G)$ in $\operatorname{Out}(G)$ are the same - $\operatorname{OFR}(G)=\operatorname{OWh}(G)$ - and so $\operatorname{OFR}(G)$ will not appear again. (For more background on these definitions see [MM96, especially page 48.)

A palindromic automorphism of a free group $F_{n}=F\left(a_{1}, \ldots, a_{n}\right)$ is an automorphism sending each generator $a_{i}$ to a palindromic word. The elementary palindromic automorphisms are those which send each $a_{i}$ to a palindrome of odd length with $a_{i}$ as its centermost letter. Lastly, the pure palindromic automorphisms are those which send each $a_{i}$ to an odd palindrome with either $a_{i}$ or $a_{i}^{-1}$ in the center. After identifying these automorphisms of $F_{n}$ with ones of $*_{i=0}^{n} \mathbb{Z}_{2}$, we are able to prove:

Theorem C. If $F_{n}$ is a free group of rank $n$, then the Euler characteristics of the elementary palindromic automorphism group of $F_{n}$, the pure palindromic automorphism group of $F_{n}$, and the palindromic automorphism group of $F_{n}$ are:

$$
\begin{aligned}
\chi\left(E \Pi A\left(F_{n}\right)\right) & =(1-n)^{n-1}, \\
\chi\left(P \Pi A\left(F_{n}\right)\right) & =\frac{(1-n)^{n-1}}{2^{n}}, \\
\chi\left(\Pi A\left(F_{n}\right)\right) & =\frac{(1-n)^{n-1}}{2^{n} \cdot n !} .
\end{aligned}
$$

Even though our stated theorems are related to group cohomology, the underlying arguments are primarily combinatorial. In the next section we give background information on polynomial identities and a particularly useful identity relating a multi-variable polynomial with the number of rooted trees and planted forests. Section 3 is the heart of the combinatorial work, where we use the polynomial identities 
to establish a number of partition identities and ultimately an identity involving a summation of particular weights over the set of hypertrees (Theorem 3.11). Then in Section 4, the notion of a Euler characteristic of a group is reviewed, the space constructed by McCullough and Miller is outlined, and an approach to calculating the Euler characteristic of $\mathrm{OWH}(G)$ is sketched. In Section [5, the hypertree identity is used to complete the calculation, and we complete the proofs of Theorems A and $\mathrm{B}$, as well as give some concrete examples. In Section 6 we turn to the group of palindromic automorphisms of $F_{n}$ and establish Theorem C.

Although the current ordering of the sections in the paper presents results in the order in which they are proven, an alternate - possibly more conceptual and motivated - ordering would be

$$
\S 1 \Rightarrow \S 4 \Rightarrow \S 5 \Rightarrow \S 6 \Rightarrow \S 3 \Rightarrow \S 2 \text {. }
$$

Sections 4-6 show how the Euler characteristic calculations can be derived from a particular weighted sum over hypertrees. This hypertree identity is reduced to two weighted sums over the set of partitions in $\S 3$, and finally, the necessary partition identities are established using a polynomial identity described in $\S 2$.

\section{Polynomials and trees}

In this section we record some well-known polynomial identities that we need later in the article. The first two identities were discovered by Niels Abel. In order to make them easier to state we introduce the following function.

Definition 2.1 (An interesting basis). For each non-negative integer $k$ let $h_{k}(x)$ denote the polynomial $x(x+k)^{k-1}$. Note that when $k=0$ the expression $x(x+0)^{-1}$ is not quite the same as the constant polynomial 1 since it is undefined at $x=0$, but this situation can be remedied by explicitly defining $h_{0}(x)=1$. The polynomials $\left\{h_{k}(x)\right\}_{k \geq 0}$ form a basis for the ring of polynomials in $x$, because the degree of $h_{k}(x)$ is $k$ and thus there is exactly one polynomial of each degree. As a consequence, the "monomials" of the form $h_{k_{1}}\left(x_{1}\right) \cdot h_{k_{2}}\left(x_{2}\right) \cdots h_{k_{n}}\left(x_{n}\right)$ with each $k_{i} \geq 0$ form a basis for the ring of polynomials in commuting variables $x_{1}, x_{2}, \ldots, x_{n}$.

Lemma 2.2 (Abel's identities). For each non-negative integer n, the two polynomial identities listed below are valid:

$$
\begin{aligned}
(x+y+n)^{n} & =\sum_{i+j=n}\left(\begin{array}{c}
n \\
i
\end{array}\right) h_{i}(x) \cdot(y+j)^{j}, \\
h_{n}(x+y) & =\sum_{i+j=n}\left(\begin{array}{c}
n \\
i
\end{array}\right) h_{i}(x) \cdot h_{j}(y) .
\end{aligned}
$$

These identities are readily established by induction (see for example Ro84). The second identity listed has an obvious multi-variable generalization using multinomial coefficients.

Remark 2.3. The exponential generating function for $h_{n}(x)$ is the function GKP94] calls $\left(\mathcal{E}_{1}(z)\right)^{x}$. The generating function equivalent of Lemma 2.2 is the fact that $\left(\mathcal{E}_{1}(z)\right)^{x} \cdot\left(\mathcal{E}_{1}(z)\right)^{y}=\left(\mathcal{E}_{1}(z)\right)^{x+y}$.

Definition 2.4 (Trees and forests). A forest is a simplicial graph with no cycles and a tree is a connected forest. All of the trees and forests considered here have 
distinguishable vertices. In particular, if $[n]:=\{1, \ldots, n\}$, then it is a common practice to consider the trees or forests that can be described using $[n]$ as the vertex set. A tree or forest with a distinguished vertex selected in each connected component is said to be rooted or planted, respectively. In a planted forest we consider each edge as oriented away from the root in its component. The rooted degree of a vertex $i$ in a planted forest is the number of edges starting at $i$. Notice that the rooted degree of a vertex is one less than its valence unless that vertex happens to be a root, in which case the two numbers are equal. The degree sequence of a planted forest on $[n]$ is the $n$-tuple of rooted degrees $\delta_{i}$ (in order) for $i \in[n]$, and the associated degree sequence monomial is $x_{1}^{\delta_{1}} x_{2}^{\delta_{2}} \cdots x_{n}^{\delta_{n}}$. See Figure 1 for an example.

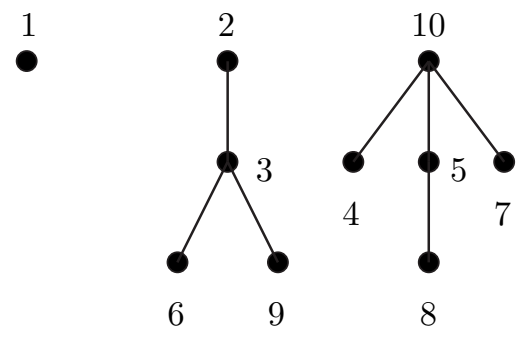

Figure 1. This planted forest on [10] has roots 1,2 and 10 . Its degree sequence is $(0,1,2,0,1,0,0,0,0,3)$ and the associated degree sequence monomial is $x_{2} x_{3}^{2} x_{5} x_{10}^{3}$.

Theorem 2.5 (A forest identity, Theorem 5.3.4 in St99]). Let $\delta=\left(\delta_{1}, \ldots, \delta_{n}\right) \in$ $\mathbb{N}^{n}$ with $\sum \delta_{i}=n-k$. The number $N(\delta)$ of planted forests $\sigma$ on the vertex set $[n]$ (necessarily with $k$ components) with ordered degree sequence $\Delta(\sigma)=\delta$ is given by

$$
N(\delta)=\left(\begin{array}{l}
n-1 \\
k-1
\end{array}\right)\left(\begin{array}{c}
n-k \\
\delta_{1}, \delta_{2}, \ldots, \delta_{n}
\end{array}\right)
$$

Equivalently,

$$
\sum_{\sigma} x_{1}^{\operatorname{deg} 1} \cdots x_{n}^{\operatorname{deg} n}=\left(\begin{array}{c}
n-1 \\
k-1
\end{array}\right)\left(x_{1}+\cdots+x_{n}\right)^{n-k},
$$

where $\sigma$ ranges over all planted forests on $[n]$ with $k$ components.

As an illustration, consider the 6 planted forests on $[3]:=\{1,2,3\}$ with exactly 2 components. Each such forest contains a single edge and a unique vertex with a non-zero degree so that the final sum in the theorem is $2\left(x_{1}+x_{2}+x_{3}\right)$ as claimed.

\section{Partitions AND hypertrees}

In this section we establish several identities involving summations over sets of partitions and hypertrees. Our Euler characteristic computations ultimately involve sums over the poset of labelled hypertrees, a poset whose internal structure is quite similar to the well-studied poset of partitions. After certain polynomial identities over the poset of partitions are established in the first subsection, we convert them into hypertree identities in the second subsection. 


$$
\chi\left(\mathrm{WH}\left(G_{1} * \cdots * G_{n}\right)\right)=\chi\left(G_{1} * \cdots * G_{n}\right)^{n-1}
$$

\subsection{Partition identities.}

Definition 3.1 (Partitions). Recall that a partition $\sigma$ of $[n]$ is a collection of pairwise disjoint subsets of $[n]$ whose union is $[n]$. These subsets (i.e. the elements of $\sigma$ ) are called blocks. The collection of all partitions of $[n]$ is denoted $\Pi_{n}$, and the subcollection of all partitions with exactly $k$ blocks is denoted $\Pi_{n}^{k}$.

Definition 3.2 (Partition sums). For each $i \in\{1, \ldots, n\}$, let $w_{i}$ be a fixed choice of a symmetric polynomial in $i$ variables. These functions can be used to define weights on the blocks of a partition as follows. Given a partition $\sigma$ of $[n]$ and an element $\tau$ in $\sigma$ of size $i$ we evaluate $w_{i}$ at the variables $x_{j}, j \in \tau$. The corresponding partition weight function is given by taking the product over all the blocks in the partition $\sigma$. The partition lattice function $f(n, k)$ is the sum of the partition weight functions over all partitions of $[n]$ with exactly $k$ blocks.

One particular symmetric polynomial occurs often enough below so as to merit a special notation. For any set $\tau \subset[n]$ define $s_{\tau}$ as the sum of the variables $x_{i}$ over all $i \in \tau$. For example, $s_{\{1,2,4\}}=x_{1}+x_{2}+x_{4}$.

Example 3.3. Let $n=3$ and $k=2$. If the symmetric polynomials $s_{\tau}$ are used as block weights, then the partition lattice function $f(3,2)$ is

$$
x_{1}\left(x_{2}+x_{3}\right)+x_{2}\left(x_{1}+x_{3}\right)+x_{3}\left(x_{1}+x_{2}\right)=2\left(x_{1} x_{2}+x_{1} x_{3}+x_{2} x_{3}\right) .
$$

Notice that it is clear from the definition that $f(n, k)$ is itself always a symmetric function on $n$ variables, but it is not at all clear whether one should expect closed form formulae for $f(n, k)$ for a particular choice of block weights. Using the forest identity from the previous section, it is relatively easy to establish at least one such summation.

Lemma 3.4 ( $1^{\text {st }}$ partition identity). The partition weight function $f(n, k)$ that weights each block $\tau$ by $\left(s_{\tau}\right)^{|\tau|-1}$ has closed form $\left(\begin{array}{c}n-1 \\ k-1\end{array}\right)\left(s_{[n]}\right)^{n-k}$. In other words,

$$
f(n, k)=\sum_{\sigma \in \Pi_{n}^{k}}\left(\prod_{\tau \in \operatorname{BLOCKS}(\sigma)}\left(s_{\tau}\right)^{|\tau|-1}\right)=\left(\begin{array}{l}
n-1 \\
k-1
\end{array}\right)\left(s_{[n]}\right)^{n-k} .
$$

Proof. Using the forest identity (Theorem 2.5), the weight on block $\tau$ is equal to a sum of degree sequence monomials for all rooted trees on the underlying set $\tau$. Replacing each block weight with this summation over rooted trees reveals that the left hand side is actually a sum of the degree sequence monomials over all planted forests with exactly $k$ components. A second use of Theorem 2.5 completes the proof.

By specializing each variable $x_{i}$ to 1 , we obtain the following result as an immediate corollary.

Corollary 3.5 ( $2^{\text {nd }}$ partition identity). The partition weight function $f(n, k)$ which weights each block $\tau$ by $|\tau|^{|\tau|-1}$ has closed form $\left(\begin{array}{c}n-1 \\ k-1\end{array}\right) n^{n-k}$. In other words,

$$
f(n, k)=\sum_{\sigma \in \Pi_{n}^{k}}\left(\prod_{\tau \in \operatorname{BLOCKS}(\sigma)}|\tau|^{|\tau|-1}\right)=\left(\begin{array}{l}
n-1 \\
k-1
\end{array}\right) n^{n-k} .
$$


A second corollary uses the alternative basis described in Definition 2.1. Recall that $h_{k}(x)$ denotes the polynomial $x(x+k)^{k-1}$ so that if $\tau=\{1,2,4,5\}$, then $h_{|\tau|-1}\left(s_{\tau}\right)=\left(x_{1}+x_{2}+x_{4}+x_{5}\right)\left(x_{1}+x_{2}+x_{4}+x_{5}+3\right)^{2}$.

Corollary 3.6 ( $3^{\text {rd }}$ partition identity). The partition weight function $f(n, k)$ which weights each block $\tau$ by $h_{|\tau|-1}\left(s_{\tau}\right)$ has closed form $\left(\begin{array}{c}n-1 \\ k-1\end{array}\right) h_{n-k}\left(s_{[n]}\right)$. In other words,

$$
f(n, k)=\sum_{\sigma \in \Pi_{n}^{k}}\left(\prod_{\tau \in \operatorname{BLOCKS}(\sigma)} h_{|\tau|-1}\left(s_{\tau}\right)\right)=\left(\begin{array}{c}
n-1 \\
k-1
\end{array}\right) h_{n-k}\left(s_{[n]}\right) .
$$

Proof. Consider the linear operator $T$ on $\mathbb{R}\left[x_{1}, \ldots, x_{n}\right]$ (viewed as an $\mathbb{R}$-vector space) that sends the monomial $x_{1}^{\delta_{1}} \cdots x_{n}^{\delta_{n}}$ to the "monomial" $h_{\delta_{1}}\left(x_{1}\right) \cdots h_{\delta_{n}}\left(x_{n}\right)$. If both sides of the equation in the statement of Lemma 3.4 are expanded using the usual binomial theorem, then the fact that the coefficients of the corresponding monomials on each side are equal is essentially the content of the first part of the forest identity from Theorem 2.5. If each monomial on each side is now replaced with the corresponding $h$-monomial (i.e. its image under $T$ ) — without changing the coefficients used - then the $h$-version of the binomial theorem (Lemma 2.2) can be used to recombine each side so as to establish the equality claimed above.

\subsection{Hypertree identities.}

Definition 3.7 (Hypertrees and hyperforests). A hypergraph $\Gamma$ is an ordered pair $(V, E)$ where $V$ is the set of vertices and $E$ is a collection of subsets of $V$-called hyperedges - each containing at least two elements. A walk in a hypergraph $\Gamma$ is a sequence $v_{0}, e_{1}, v_{1}, \ldots, v_{n-1}, e_{n}, v_{n}$ where for all $i, v_{i} \in V, e_{i} \in E$ and for each $e_{i},\left\{v_{i-1}, v_{i}\right\} \subset e_{i}$. A hypergraph is connected if every pair of vertices is joined by a walk. A simple cycle is a walk that contains at least two edges, all of the $e_{i}$ are distinct and all of the $v_{i}$ are distinct except $v_{0}=v_{n}$. A hypergraph with no simple cycles is a hyperforest, and a connected hyperforest is a hypertree. Note that the no simple cycle condition implies that distinct edges in $\Gamma$ have at most one vertex in common. All of the hypertrees and hyperforests considered here have distinguishable vertices. In particular, it is common practice to mainly consider the hypertrees or hyperforests that can be described using $[n]$ as the vertex set. Examples of hypertrees on $[n]$ are shown in Figures 2 and 3 , where hyperedges are indicated by polygons.

Many of the concepts previously defined for trees and forests have natural extensions to hypertrees and hyperforests. For example, a hypertree or hyperforest with a distinguished vertex selected in each connected component is said to be rooted or planted, respectively. In a planted hyperforest it is common practice to consider each hyperedge to be "oriented" by gravity, away from the root of the component. In this case the "orientation" of a hyperedge distinguishes between the unique vertex closest to the root and all of the remaining vertices in the hyperedge. We say that the closest vertex is where the hyperedge starts, and the others are where it ends. The rooted degree of a vertex $i$ in a planted hyperforest is the number of hyperedges starting at $i$. Notice that the rooted degree of a vertex is one less than its valence (i.e. the number of hyperedges that contain $i$ ) unless that vertex happens to be a root, in which case the two numbers are equal. Finally, to establish notation, let $\mathrm{PHF}_{n}^{k}$ denote the set of all planted hyperforests on $[n]$ with exactly 
$k$ components and let $\mathrm{RHT}_{n}\left(=\mathrm{PHF}_{n}^{1}\right)$ denote the set of all rooted hypertrees on $[n]$.

We establish a pair of identities involving summations of symmetric polynomials over planted hyperforests and (unrooted) hypertrees, respectively. We begin by defining a particular weight function for rooted hyperforests that is similar to the block weights and partition lattice functions considered above.

Definition 3.8 (Weight of a planted hyperforest). Let $\tau$ be a planted hyperforest. Define the weight of an edge in $\tau$ as $(e-1)^{e-2}$ where $e$ is its size. Define the weight of the vertex $i$ as $x_{i}^{\operatorname{deg} i}$ where $\operatorname{deg} i$ is its rooted degree. The weight of $\tau$ is the product of all of its edge and vertex weights. Thus,

$$
\operatorname{Weight}(\tau)=\left(\prod_{e \in \operatorname{EdgEStzes}(\tau)}(e-1)^{e-2}\right) \cdot\left(\prod_{i \in \operatorname{Vertices}(\tau)} x_{i}^{\operatorname{deg} i}\right) .
$$

The main combinatorial result in this article is an assertion that the sum over all planted hyperforests of their weights is equal to a very simple symmetric function. This formula is the key step in establishing Theorem $\mathrm{A}$.

Theorem 3.9 (A hyperforest identity). When the weight of a planted hyperforest is defined as above, the sum of these weights over all planted hyperforests on $[n]$ with exactly $k$ components is a function of the linear symmetric function. More specifically,

$$
\sum_{\tau \in \mathrm{PHF}_{n}^{k}} \operatorname{WeIGHT}(\tau)=\left(\begin{array}{l}
n-1 \\
k-1
\end{array}\right) h_{n-k}\left(s_{[n]}\right)
$$

which specializes to

$$
\sum_{\tau \in \mathrm{RHT}_{n+1}} \operatorname{WeIGHT}(\tau)=h_{n}\left(s_{[n+1]}\right)=s_{[n+1]}\left(s_{[n+1]}+n\right)^{n-1}
$$

when summing these weights over rooted hypertrees on $[n+1]$.

Proof. The proof is by induction on the ordered pairs $(n, k)$, ordered lexicographically. Since the base case $(1,1)$ is easily checked, suppose that the result has been established for all ordered pairs less than $(n, k)$. The inductive step splits into two cases depending on whether $k=1$ or $k>1$. When $k>1$, then the sum on the lefthand side can be rearranged according to the partition of $[n]$ determined by the connected components of the hyperforest under consideration. Once a partition $\sigma$ in $\Pi_{n}^{k}$ has been fixed, the hyperforests associated to it are found by picking a rooted hypertree on each block of $\sigma$. Because these choices can be made independent of one another, and because the weight of a planted hyperforest is the product of the weights of its rooted hypertrees, the sum of the hyperforest weights over the hyperforests associated with this particular partition is a product of sums that only focus on one block of $\sigma$ at a time. In addition, if $\tau$ is a block of $\sigma$, then the inductive hypothesis can be applied to the sum of rooted hypertree weights over each of all rooted hypertrees on $\tau$ since the size of $\tau$ must be strictly less than $n$. Thus we have

$$
\sum_{\mu \in \operatorname{PHF}_{n}^{k}} \operatorname{Weight}(\mu)=\sum_{\sigma \in \Pi_{n}^{k}} \prod_{\tau \in \operatorname{BLOCKS} \sigma} h_{|\tau|-1}\left(s_{\tau}\right),
$$

which is equal to $\left(\begin{array}{c}n-1 \\ k-1\end{array}\right) h_{n-k}\left(s_{[n]}\right)$ by the $3^{r d}$ partition identity, Corollary 3.6 
When $k=1$, the sum on the lefthand side is only over rooted hypertrees and the approach is slightly different. In order for the algebraic manipulations to be easier to follow, we consider the case $(n+1,1)$ rather than $(n, 1)$. If $\mu$ is a rooted hypertree on $[n+1]$, then there is a planted hyperforest on $n$ vertices associated with $\mu$ which can be found by removing its root and all of the hyperedges containing it. The resulting components are naturally rooted by selecting the unique vertex in each that was in a common hyperedge with the original root. (See Figure 2,)

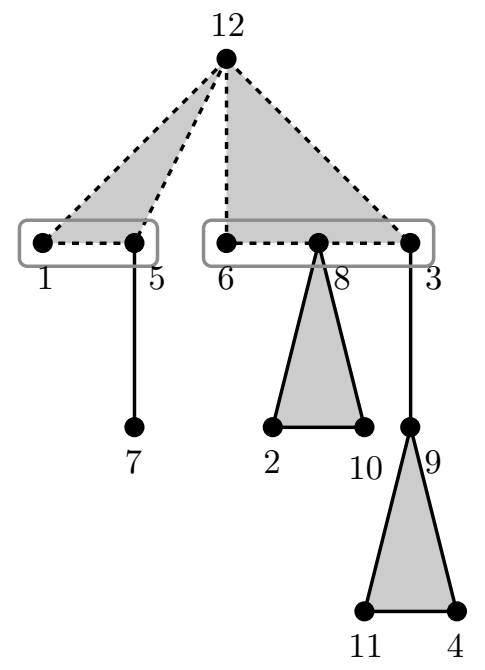

Figure 2. A rooted hypertree on [12] with its associated planted hyperforest on [11]. One can pass from the hyperforest back to the hypertree using the partition of $\{1,3,5,6,8\}$ indicated by the lightly colored boxes.

As in the previous case, we proceed by rearranging a sum and applying the inductive hypothesis. Consider the portion of the lefthand side of equation (3) that only sums over those rooted hypertrees on $[n+1]$ that have the vertex $n+1$ as their root, the root has rooted degree exactly $i$, and the number of connected components in the planted forest on $[n]$ found by removing the root and all its hyperedges is exactly $k$. The collection of all rooted hypertrees having all three properties can be found as follows. First, pick an arbitrary planted forest $\mu$ with exactly $k$ components on the vertex set $[n]$. Next, pick a partition $\sigma$ of the $k$ roots of $\mu$ that has exactly $i$ blocks. Finally, for each block in $\sigma$, add a hyperedge containing the appropriate roots of $\mu$ together with the vertex $n+1$. (This process is illustrated in Figure 2 where $k=5$ and the two blocks of the partition are indicated by the lightly colored loops.) Because of the independence of the choices involved, the sum of the hypertree weights over this restricted set of rooted hypertrees can be rewritten as follows:

$$
\left(\sum_{\sigma \in \Pi_{k}^{i}} \prod_{\tau \in \operatorname{BLOCKS}(\sigma)}|\tau|^{|\tau|-1}\right)\left(\sum_{\mu \in \operatorname{PHF}_{n}^{k}} \operatorname{Weight}(\mu)\right) x_{n+1}^{i} .
$$

The first factor is the contribution of the edge weights of the hyperedges containing the root (there is a slight shift since the size of the block in $\sigma$ is one less than the 
size of the edge containing the root), the third factor is the contribution of the vertex weight of the root itself, and the second factor is the contribution of all of the other vertex and edge weights combined. By the $2^{\text {nd }}$ partition identity, from Corollary 3.5. the first factor is equal to $\left(\begin{array}{c}k-1 \\ i-1\end{array}\right) k^{k-i}$, and by the inductive hypothesis the second factor is equal to $\left(\begin{array}{c}n-1 \\ k-1\end{array}\right) h_{n-k}\left(s_{[n]}\right)$. Using the fact that

$$
\left(\begin{array}{l}
k-1 \\
i-1
\end{array}\right)\left(\begin{array}{l}
n-1 \\
k-1
\end{array}\right)=\left(\begin{array}{c}
n-1 \\
i-1, k-i, n-k
\end{array}\right)=\left(\begin{array}{c}
n-1 \\
i-1
\end{array}\right)\left(\begin{array}{l}
n-i \\
k-i
\end{array}\right)
$$

the sum of these weights can be rewritten as

$$
\left(\begin{array}{c}
n-1 \\
i-1
\end{array}\right)\left(\begin{array}{c}
n-i \\
k-i
\end{array}\right) k^{k-i} h_{n-k}\left(s_{[n]}\right) x_{n+1}^{i} .
$$

A similar formula holds when any other vertex is selected as the root.

Finally, it only remains to eliminate the dependence of this sum on $k, i$, and the choice of a root vertex. One form of Abel's identity, equation (1), is

$$
\sum_{k}\left(\begin{array}{l}
n-i \\
k-i
\end{array}\right) h_{n-k}\left(s_{[n]}\right) k^{k-i}=\left(s_{[n]}+n\right)^{n-i},
$$

where we have substituted $s_{[n]}$ for $x, i$ for $y, n-i$ for $n, n-k$ for $i$, and $k-i$ for $j$. Thus, summing over all possible values of $k$ we find that the sum of the weights over all hypertrees with root vertex $n+1$ and root degree $i$ is equal to $\left(\begin{array}{c}n-1 \\ i-1\end{array}\right)\left(s_{[n]}+n\right)^{n-i} x_{n+1}^{i}$. Summing over all possible values of $i$, factoring out a single $x_{n+1}$ and applying the binomial theorem we find that the sum of the weights of all hypertrees with root $n+1$ is $x_{n+1}\left(s_{[n]}+n+x_{n+1}\right)^{n-1}=x_{n+1}\left(s_{[n+1]}+n\right)^{n-1}$. Finally, summing over the possible choices of the root vertex gives $s_{[n+1]}\left(s_{[n+1]}+n\right)^{n-1}$ which is precisely $h_{n}\left(s_{[n+1]}\right)$ as desired. This completes the inductive step when $k=1$ and the proof.

As is often the case, we can use the identity established for rooted hypertrees to establish a similar identity for unrooted hypertrees.

Definition 3.10 (Weight of a hyperforest). If $\tau$ is an (unplanted) hyperforest, then the weight of an edge in $\tau$ is as before (i.e. $(e-1)^{e-2}$ where $e$ is its size), but the weight of the vertex $i$ is now $x_{i}^{\operatorname{val}(i)-1}$ where $\operatorname{val}(i)$ is its valence. The edge weight of $\tau$ is the product of the weights of its edges,

$$
\operatorname{E-Weight}(\tau)=\prod_{e \in \operatorname{EdGESizes}(\tau)}(e-1)^{(e-2)} .
$$

The vertex weight of $\tau$ is the product of the weights of its vertices,

$$
\operatorname{V-Weight}(\tau)=x_{1}^{\operatorname{val}(1)-1} x_{2}^{\operatorname{val}(2)-1} \cdots x_{n}^{\mathrm{val}(n)-1},
$$

which is closely related to the "degree sequence monomial" of a planted forest. The weight of $\tau$ is

$$
\operatorname{Weight}(\tau)=\operatorname{E-} \operatorname{Weight}(\tau) \cdot \operatorname{V}-\operatorname{Weight}(\tau),
$$

so it is still the product of $\tau$ 's edge and vertex weights.

Notice that these definitions almost completely agree with those given in Definition 3.8. In particular, if $\tau$ is a rooted hypertree on $[n]$ rooted at vertex $i$, then its weight when viewed as a rooted hypertree is $x_{i}$ times its weight when viewed as an unrooted hypertree. This is because $\operatorname{val}(j)-1=\operatorname{deg} j$ except at the root. As a 
consequence, if $\tau$ is a hypertree and $\tau_{i}$ is the hypertree $\tau$ with root vertex $i$, then the sum of the rooted weights of the $\tau_{i}$ is $s_{[n]}$ times the weight of $\tau$.

Theorem 3.11 (A hypertree identity). When the weight of a hypertree is defined as above, the sum of these weights over all hypertrees on $[n+1]$ is a function of the linear symmetric function. More specifically,

$$
\sum_{\tau \in \mathrm{HT}_{n+1}} \operatorname{WeIGHT}(\tau)=\left(s_{[n+1]}+n\right)^{n-1} .
$$

Proof. By the remark made above, the sum of the rooted weights over all rooted hypertrees on $[n+1]$ is equal to $s_{[n+1]}$ times the sum of the weights over all (unrooted) hypertrees on $[n+1]$. The formula now follows from the second formula in the statement of Theorem 3.9 .

Here are two small examples that illustrate the equality.

Example 3.12 (Low dimensional cases). When $n=2$, the poset $\mathrm{HT}_{3}$ has 4 elements and the 4 terms on the lefthand side are $1 \cdot x_{1}, 1 \cdot x_{2}, 1 \cdot x_{3}$ and $2 \cdot 1$. Thus the total is $x_{1}+x_{2}+x_{3}+2$ as predicted by the righthand side.

There are 4 types of hypertrees in $\mathrm{HT}_{4}$, and 29 elements total in the poset. There is 1 element consisting of a single hyperedge (Type A); 12 elements consisting of 2 hyperedges (Type B); 12 elements that are simplicial arcs (Type C); and 4 elements that are simplicial trees containing a central trivalent vertex (Type D). (These are drawn in Figure 3 of MM04.) Starting on the lefthand side, we have that the element of type $A$ contributes $3^{2}$, the elements of type $B$ add $6\left(x_{1}+x_{2}+x_{3}+x_{4}\right)$, the elements of type $C$ add $2\left(x_{1} x_{2}+x_{1} x_{3}+x_{1} x_{4}+x_{2} x_{3}+x_{2} x_{4}+x_{3} x_{4}\right)$ and the elements of type $D$ add $x_{1}^{2}+x_{2}^{2}+x_{3}^{2}+x_{4}^{2}$. So for $n=3$ the total is $\left(x_{1}+x_{2}+x_{3}+x_{4}+3\right)^{2}$ as expected.

\section{Euler, McCullough And Miller}

Our Euler characteristic results follow from a computation using the action of outer Whitehead automorphism groups on certain contractible complexes introduced by McCullough and Miller [MM96]. We recall relevant facts about Euler characteristics of groups and about these McCullough-Miller complexes.

4.1. Euler characteristics. Because the homotopy type of an aspherical complex is determined by its fundamental group, it makes sense to define the Euler characteristic of a group $G$ as the Euler characteristic of any finite $K(G, 1)$. However, a group does not have to be of finite type to have a well defined Euler characteristic. A group $G$ is of finite homological type if $G$ has finite vcd, and for every $i$ and every $G$-module $M$ that is finitely generated as an abelian group, $H_{i}(G, M)$ is finitely generated. This holds, for example, if $G$ is VFL, that is, has a finite index subgroup $H$ where $\mathbb{Z}$ admits a finite free resolution as a trivial $\mathbb{Z} H$-module. The Euler characteristic can be defined for any group of finite homological type.

To complete the proofs of Theorems $[\mathrm{A}, \mathrm{B}$ and $\mathrm{C}$, we need some basic facts about Euler characteristics of groups. The following results are parts $(b)$ and $(d)$ of Theorem 7.3 in Chapter IX of [Br94.

Theorem 4.1. Let $G$ be a group of finite homological type.

1. If $H$ is a subgroup of finite index in $G$, then $\chi(H)=\chi(G) \cdot[G: H]$. 


$$
\chi\left(\mathrm{WH}\left(G_{1} * \cdots * G_{n}\right)\right)=\chi\left(G_{1} * \cdots * G_{n}\right)^{n-1}
$$

2. If $1 \rightarrow K \rightarrow G \rightarrow Q \rightarrow 1$ is a short exact sequence of groups (with $K$ and $Q$ of finite homological type), then $\chi(G)=\chi(K) \cdot \chi(Q)$.

Our approach to computing the Euler characteristics of Whitehead automorphism groups is via the standard formula for equivariant Euler characteristics (see [Br74] and $\S I X .7$ of [Br94]).

Theorem 4.2. Let $X$ be a cocompact, contractible $G$-complex, and let $\mathcal{E}$ be a set of representatives of the cells of $X \bmod G$. Then

$$
\chi(G)=\sum_{\sigma \in \mathcal{E}}(-1)^{\operatorname{dim}(\sigma)} \chi\left(G_{\sigma}\right) .
$$

In particular, if $G=*_{i=1}^{n} G_{i}$ is a free product of groups of finite homological type, then the associated action of $G$ on its Bass-Serre tree shows that

$$
\chi(G)=\chi\left(G_{1}\right)+\cdots+\chi\left(G_{n}\right)-(n-1) .
$$

Euler characteristics of groups have been computed in a number of interesting cases. For example, the Euler characteristics of many arithmetic groups can be expressed in terms of zeta-functions, an interesting example being

$$
\chi\left(\mathrm{SL}_{2}(\mathbb{Z}[1 / p])\right)=\frac{p-1}{12}
$$

(see the survey [Se79]). The Euler characteristics of mapping class groups of surfaces have also been explicitly computed ([HZ86, Pe88]). In general Euler characteristics of families of groups do not have tidy formulas. For example, a generating function for $\chi\left(\operatorname{OUT}\left(F_{n}\right)\right)$ is presented in [SV87, but the situation is sufficiently complicated that no closed form solution is given. Computer computations are used to find $\chi\left(\operatorname{Out}\left(F_{n}\right)\right)$ up to $n=12$, where

$$
\chi\left(\operatorname{OuT}\left(F_{12}\right)\right)=-\frac{375393773534736899347}{2191186722816000} .
$$

For further information on Euler characteristics of groups read Chapter IX of [Br94].

4.2. McCullough-Miller complexes. Given a free product $G=G_{1} * \cdots * G_{n}$, McCullough and Miller construct an $\mathrm{OWH}(G)$ complex that can be viewed as a space of certain actions of $G$ on simplicial trees. At the heart of their construction is the poset of hypertrees.

Definition 4.3 (The hypertree poset). The set of all hypertrees on $V=[n]$ forms a poset, $\mathrm{HT}_{n}$, where $\tau \leq \tau^{\prime}$ if each edge of $\tau^{\prime}$ is contained in an edge of $\tau$. We write $\tau<\tau^{\prime}$ if $\tau \leq \tau^{\prime}$ but $\tau \neq \tau^{\prime}$. The poset $\mathrm{HT}_{n}$ is a graded poset, and the hypertrees at height $i$ are precisely those hypertrees with $i+1$ edges. Notice that $\mathrm{HT}_{n}$ contains a unique minimal element (with height 0 ) that has only a single edge containing all of $[n]$. This is the nuclear vertex and we denote it by $\hat{0}$. In Figure 3 we show the Hasse diagram for the interval $[\hat{0}, \tau] \subset \mathrm{HT}_{5}$ where $\tau$ is a maximal element of height 3. For convenience we have used convex polygons (edges, triangles, squares, etc.) to indicate the hyperedges. Since $\mathrm{HT}_{5}$ contains 311 elements, 125 of which are maximal elements, we do not show the entire poset. If $\tau \in \mathrm{HT}_{n}$ let $\mathrm{HT}_{n, \geq \tau}$ and $\mathrm{HT}_{n,>\tau}$ denote the subposets induced by elements which are above, or strictly above, $\tau$. 


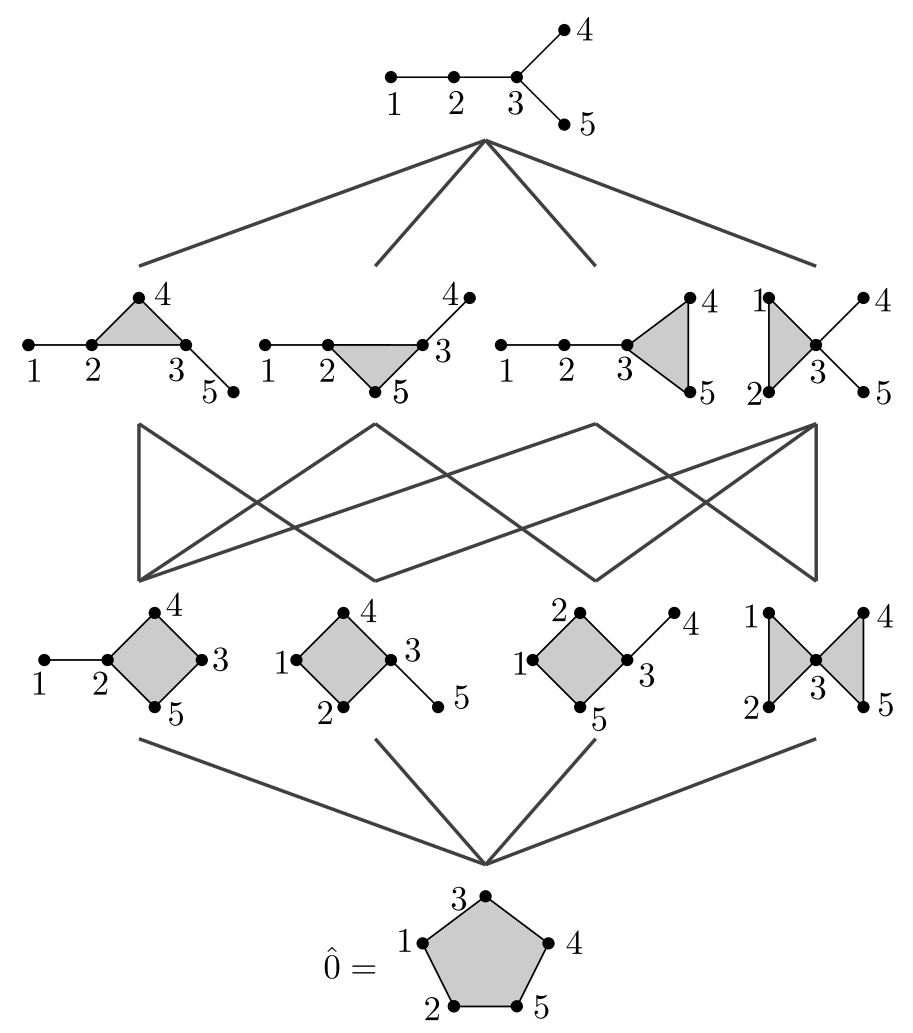

Figure 3. The Hasse diagram of the closed interval between 0 and one of the 125 elements of maximal rank in $\mathrm{HT}_{5}$.

An easy induction establishes the following well-known fact.

Lemma 4.4. For any hypertree $\tau$,

$$
\operatorname{Height}(\tau)=\# E(\tau)-1=\sum_{v \in V(\tau)}(\operatorname{val}(v)-1),
$$

where $\operatorname{val}(v)$ is the valence of $v$, i.e. the number of hyperedges containing $v$.

We refer to the value multiply described in Lemma 4.4 as the rank of the hypertree $\tau$, and denote it $\operatorname{rank}(\tau)$.

The main result of [MM96] can be formulated as follows.

Theorem 4.5. Given $G=G_{1} * \cdots * G_{n}$ there is a contractible $\mathrm{OWH}(G)$-complex $M M_{n}$ with $\mathrm{OWH}(G) \backslash M M_{n} \simeq\left|\mathrm{HT}_{n}\right|$.

Here $\left|\mathrm{HT}_{n}\right|$ denotes the geometric realization of $\mathrm{HT}_{n}$. The reader wishing to understand the connection between this description and the original description as given in MM96] can consult MM04. Geometrically, the complex $\mathrm{MM}_{n}$ contains an imbedded copy of $\left|\mathrm{HT}_{n}\right|$ that projects isomorphically to the quotient under the action, and McCullough and Miller give a clean description of simplex stabilizers. 
Theorem 4.6. The stabilizer of $\tau \in\left|\mathrm{HT}_{n}\right|$ under the action of $\mathrm{OWH}(G)$ is

$$
\operatorname{Stab}(\tau)=\bigoplus_{i=1}^{n}\left(G_{i}\right)^{\operatorname{val}(i)-1}
$$

Further, if $\tau<\tau^{\prime}<\cdots<\tau^{\prime \prime}$ is the chain corresponding to a simplex $\sigma \in\left|\mathrm{HT}_{n}\right|$, then $\operatorname{Stab}(\sigma)=\operatorname{Stab}(\tau)$.

If the $G_{i}$ are all of finite homological type, then the simplex stabilizers in $\mathrm{MM}_{n}$ under the action of $\mathrm{OWH}(G)$ are of finite homological type. Using the fact that $\mathrm{MM}_{n}$ is a finite dimensional, contractible, cocompact $\mathrm{OWH}(G)$-complex, we get:

Corollary 4.7. If $G=*_{i=1}^{n} G_{i}$, and each of the $G_{i}$ is of finite homological type, then $\mathrm{OWH}(G)$ is of finite homological type.

Remark 4.8. Even if $G$ is of finite homological type, $\operatorname{Aut}(G)$ may not be. For example, the Baumslag-Solitar group $\operatorname{BS}(2,4)$ is of finite type, yet $\operatorname{Aut}(\operatorname{BS}(2,4))$ is not finitely generated CL83. Corollary 4.7 establishes that $\chi(\mathrm{OWH}(G))$ and $\chi(\mathrm{WH}(G))$ exist when $G$ is a free product of groups of finite homological type, but the example of $\operatorname{BS}(2,4)$ illustrates that in general $\chi(\operatorname{AUT}(G))$ may not exist. Thus one cannot hope to establish a result such as Theorem $B$ without some reasonable hypotheses such as "all $G_{i}$ finite."

We record a few useful facts about the combinatorics of $\mathrm{HT}_{n}$ from MM04.

Lemma 4.9. Let $\tau$ be a hypertree on the set $[n]$. The subposet $\mathrm{HT}_{n, \geq \tau}$ decomposes as a direct product of hypertree posets:

$$
\operatorname{HT}_{n, \geq \tau}=\prod_{j \in \operatorname{EdGESIzES}(\tau)} \operatorname{HT}_{j}
$$

Recall that the reduced Euler characteristic of a complex $K$ is $\widetilde{\chi}(K)=\chi(K)-1$, that is, it is the Euler characteristic based on reduced homology.

Theorem 4.10. For every $n$,

$$
\widetilde{\chi}\left(\left|\mathrm{HT}_{n+1,>0 \hat{0}}\right|\right)=(-1)^{n} n^{n-1} .
$$

Theorem 4.10 and Lemma 4.9 combine to give the following formula. The only tricky matter is establishing the sign. See [MM04, Lemma 4.6] for the details.

Corollary 4.11. The reduced Euler characteristic of $\left|\mathrm{HT}_{n,>\tau}\right|$ is

$$
\begin{aligned}
\tilde{\chi}\left(\left|\mathrm{HT}_{n,>\tau}\right|\right) & =(-1)^{n+|\operatorname{Edges}(\tau)|} \prod_{j \in \operatorname{EdGESizes}(\tau)}(j-1)^{(j-2)} \\
& =(-1)^{n+|\operatorname{Edges}(\tau)|} \cdot \operatorname{E-} \operatorname{WEight}(\tau)
\end{aligned}
$$

\section{Proofs And Computations}

In this final section we complete the proofs of Theorems $\mathrm{A}$ and $\mathrm{B}$. First, we express the Euler characteristic of $\mathrm{OWH}(G)$ in terms of a sum over hypertrees.

Lemma 5.1. Let $G=*_{i=1}^{n} G_{i}$, where each $G_{i}$ is of finite homological type. Then

$$
\chi(\mathrm{OWH}(G))=(-1)^{n} \sum_{\tau \in \mathrm{HT}_{n}}(-1)^{\operatorname{rank}(\tau)} \cdot \chi(\operatorname{Stab}(\tau)) \cdot \operatorname{E}-\mathrm{WEIGHT}(\tau) .
$$


Proof. By Theorem 4.2 we know

$$
\chi(\mathrm{OWH}(G))=\sum_{\sigma \in\left|\mathrm{HT}_{n}\right|}(-1)^{\operatorname{dim}(\sigma)} \chi(\operatorname{Stab}(\sigma)) .
$$

If $\sigma=\tau<\tau^{\prime}<\cdots<\tau^{\prime \prime}$, the stabilizer of $\sigma$ is the same as the stabilizer of $\tau$. Thus we may reorganize this summation as

$$
\begin{aligned}
\chi(\mathrm{OWH}(G)) & =\sum_{\tau \in \mathrm{HT}_{n}}\left\{\chi(\operatorname{Stab}(\tau)) \sum_{\sigma \in\left|\mathrm{HT}_{n, \geq \tau}\right|}(-1)^{\operatorname{dim}(\sigma)}\right\} \\
& =\sum_{\tau \in \mathrm{HT}_{n}}\left\{\chi(\operatorname{Stab}(\tau))\left(1-\sum_{\sigma \in\left|\mathrm{HT}_{n,>\tau}\right|}(-1)^{\operatorname{dim}(\sigma)}\right)\right\} \\
& =\sum_{\tau \in \mathrm{HT}_{n}} \chi(\operatorname{Stab}(\tau))\left(-\tilde{\chi}\left(\left|\mathrm{HT}_{n,>\tau}\right|\right)\right) .
\end{aligned}
$$

But up to sign, the reduced Euler characteristic of $\left|\mathrm{HT}_{n,>\tau}\right|$ is just $\operatorname{E-WeIGHT}(\tau)$ (Corollary 4.11). Inserting the formula from Corollary 4.11 we get

$$
\begin{aligned}
\chi(\mathrm{OWH}(G)) & =\sum_{\tau \in \mathrm{HT}_{n}} \chi(\operatorname{Stab}(\tau))\left((-1)^{n+1+|\operatorname{Edges}(\tau)|} \cdot \operatorname{E-Weight}(\tau)\right) \\
& =(-1)^{n} \sum_{\tau \in \mathrm{HT}_{n}} \chi(\operatorname{Stab}(\tau)) \cdot(-1)^{\operatorname{rank}(\tau)} \cdot \operatorname{E-Weight}(\tau),
\end{aligned}
$$

which is the formula claimed in the statement of the lemma.

Theorem $\mathrm{A}$ is now essentially established. Exploiting Corollary 4.7 we can give it a slightly stronger phrasing.

Theorem A. If $G=G_{1} * \cdots * G_{n}$ is a free product of groups of finite homological type, then the Euler characteristic of the group of outer Whitehead automorphisms is $\chi(\mathrm{OWH}(G))=\chi(G)^{n-2}$. As a consequence, the Euler characteristic of the group of Whitehead automorphisms is $\chi(\mathrm{WH}(G))=\chi(G)^{n-1}$.

Proof. Because $\operatorname{Stab}(\tau)=\bigoplus_{i=1}^{n}\left(G_{i}\right)^{\operatorname{val}(i)-1}$ by Theorem 4.6, and using the equivalence of the multiple definitions of rank given in Lemma 4.4, we see that

$$
\chi(\operatorname{Stab}(\tau)) \cdot(-1)^{\operatorname{rank}(\tau)}=\prod\left(-\chi\left(G_{i}\right)\right)^{\operatorname{val}(i)-1} .
$$

Therefore we can rewrite the formula from Lemma 5.1 as

$$
\chi(\mathrm{OWH}(G))=(-1)^{n} \sum_{\tau \in \mathrm{HT}_{n}}\left[\prod\left(-\chi\left(G_{i}\right)\right)^{\operatorname{val}(i)-1}\right] \cdot \operatorname{E-WeIGHT}(\tau) .
$$

Thus - aside from the $(-1)^{n}$ term - the Euler characteristic of $\mathrm{OWH}(G)$ is given by the left side of hypertree identity (5), with " $-\chi\left(G_{i}\right)$ " being substituted for the variable " $x_{i}$ ". Making the same substitution on the right side of the hypertree identity and reinserting the $(-1)^{n}$ term shows that

$$
\begin{aligned}
\chi(\mathrm{OWH}(G)) & =(-1)^{n}\left(-\chi\left(G_{1}\right)-\chi\left(G_{2}\right)-\cdots-\chi\left(G_{n}\right)+(n-1)\right)^{n-2} \\
& =(-1)^{n}(-\chi(G))^{n-2}=\chi(G)^{n-2} .
\end{aligned}
$$

Thus we have the formula for the group of outer Whitehead automorphisms. 


$$
\chi\left(\mathrm{WH}\left(G_{1} * \cdots * G_{n}\right)\right)=\chi\left(G_{1} * \cdots * G_{n}\right)^{n-1}
$$

Item 2 of Theorem 4.1 applied to the short exact sequence

$$
1 \rightarrow G \rightarrow \mathrm{WH}(G) \rightarrow \mathrm{OWH}(G) \rightarrow 1
$$

shows that $\chi(\mathrm{WH}(G))=\chi(G) \cdot \chi(\mathrm{OWH}(G))=\chi(G)^{n-1}$.

Theorem B. If $G=G_{1} * \cdots * G_{n}$ is a free product of finite groups and $\Omega \subset \Sigma_{n}$ is the subgroup of the symmetric group given by permuting isomorphic factors $G_{i}$, then the Euler characteristics of the Fouxe-Rabinovitch automorphism group of $G$, the full automorphism group of $G$ and the outer automorphism group of $G$ are as follows:

$$
\begin{aligned}
\chi(\operatorname{FR}(G)) & =\chi(G)^{n-1} \prod_{i=1}^{n}\left|\operatorname{InN}\left(G_{i}\right)\right|, \\
\chi(\operatorname{Aut}(G)) & =\chi(G)^{n-1}|\Omega|^{-1} \prod_{i=1}^{n}\left|\operatorname{Out}\left(G_{i}\right)\right|^{-1}, \\
\chi(\operatorname{Out}(G)) & =\chi(G)^{n-2}|\Omega|^{-1} \prod_{i=1}^{n}\left|\operatorname{Out}\left(G_{i}\right)\right|^{-1} .
\end{aligned}
$$

Proof. The identities listed above are derived from Theorem $\mathrm{A}$ using finite-index subgroups and short exact sequences. To calculate $\chi(\operatorname{FR}(G))$ we use the fact that $\operatorname{FR}(G)$ is a finite-index subgroup of $\mathrm{WH}(G)$. Recall that if each $G_{i}$ is finite, then $\operatorname{Aut}(G)=\operatorname{\Sigma Aut}(G)$ and so $\operatorname{WH}(G)$ is the kernel of the map $f: \operatorname{Aut}(G) \rightarrow$ $\operatorname{Out}\left(\prod_{i} G_{i}\right)$ and $\operatorname{FR}(G)$ is the kernel of the map $g: \operatorname{Aut}(G) \rightarrow \operatorname{Aut}\left(\prod_{i} G_{i}\right)$. Moreover, the map $f=h \circ g$ where $h$ is the map $\operatorname{Aut}\left(\prod_{i} G_{i}\right) \rightarrow \operatorname{OUT}\left(\prod_{i} G_{i}\right)$. Since the kernel of $h$ is $\prod_{i} \operatorname{INN}\left(G_{i}\right)$, the index of $\operatorname{FR}(G)$ in $\operatorname{WH}(G)$ is $\prod_{i}\left|\operatorname{INN}\left(G_{i}\right)\right|$. This index is finite when each $G_{i}$ is finite, and the first identity follows by item 1 of Theorem 4.1

To calculate $\chi(\operatorname{Aut}(G))$ we use the decomposition of $\operatorname{Aut}(G)$ noted by McCullough and Miller [MM96, p. 48]:

$$
\operatorname{Aut}(G)=F R(G) \rtimes\left(\left(\prod_{i} \operatorname{Aut}\left(G_{i}\right)\right) \rtimes \Omega\right) .
$$

From this decomposition it is clear that the size of $g(\operatorname{AUT}(G))$-and the index of $\operatorname{FR}(G)$ in $\operatorname{Aut}(G)$-is $|\Omega| \prod_{i}\left|\operatorname{Aut}\left(G_{i}\right)\right|$. Dividing by the index of $\operatorname{FR}(G)$ in $\mathrm{WH}(G)$ we see that the index of $\mathrm{WH}(G)$ in $\operatorname{Aut}(G)$ is $|\Omega| \prod_{i}\left|\operatorname{Out}\left(G_{i}\right)\right|$. This index is finite and the second identity follows, again by item 1 of Theorem 4.1 . Finally, from the short exact sequence $1 \rightarrow G \rightarrow \operatorname{AuT}(G) \rightarrow \operatorname{OuT}(G) \rightarrow 1$ we find that $\chi(\operatorname{OUT}(G))=\chi(G)^{-1} \chi(\operatorname{AUT}(G))$ by item 2 of Theorem 4.1 which completes the proof.

Example 5.2 (The factors are all finite cyclic). When $G$ is a free product of finite cyclic groups, the values listed in Theorem $\mathrm{B}$ are particularly easy to compute. If $G_{i}$ is finite cyclic with order $g_{i}$, then $\chi\left(G_{i}\right)=\left(g_{i}\right)^{-1}$ and $\chi(G)=\left(\sum_{i}\left(g_{i}\right)^{-1}-\right.$ $n+1)$. Moreover, $G_{i}$ abelian implies $\left|\operatorname{INN}\left(G_{i}\right)\right|=1$ and $G_{i}$ cyclic means that $\left|\operatorname{Aut}\left(G_{i}\right)\right|=\left|\operatorname{Out}\left(G_{i}\right)\right|=\phi\left(g_{i}\right)$ where $\phi$ is Euler's totient function. Recall that $\phi$ can be defined be setting $\phi\left(p^{k}\right)=p^{k}-p^{k-1}$ for all prime powers and then extending it by $\phi(m \cdot n)=\phi(m) \cdot \phi(n)$ wherever $m$ and $n$ are relatively prime. Finally, $\Omega$ is simply a product of symmetric groups whose indices count the number of times each particular value occurs among the $g_{i}$. As a concrete illustration, when 
$G=\mathbb{Z}_{2} * \mathbb{Z}_{2} * \mathbb{Z}_{2} * \mathbb{Z}_{2} * \mathbb{Z}_{3} * \mathbb{Z}_{3} * \mathbb{Z}_{3}$, then $\chi(G)=\left(\frac{4}{2}+\frac{3}{3}-7+1\right)=-3,|\Omega|=(4 ! \cdot 3 !)$, $\phi(2)=1$ and $\phi(3)=2$. Thus $\chi(\operatorname{Aut}(G))=\frac{(-3)^{6}}{4 ! 3 !}\left(\frac{1}{1}\right)^{4}\left(\frac{1}{2}\right)^{3}=\frac{81}{128}$.

Example 5.3 (The factors are all infinite cyclic). If each $G_{i} \simeq \mathbb{Z}$, then $\mathrm{WH}\left(*_{i=1}^{n} G_{i}\right)$ is the subgroup of $\operatorname{AUT}\left(F_{n}\right)$ called the pure symmetric automorphism group, commonly denoted $P \Sigma_{n}$. The Euler characteristic of this group was computed in MM04, but the answer also follows from Theorem A. Using the fact that $\chi\left(F_{n}\right)=$ $1-n$ one gets

$$
\chi(\mathrm{WH}(\underbrace{\mathbb{Z} * \cdots * \mathbb{Z}}_{n \text { free factors }}))=\chi\left(P \Sigma_{n}\right)=(1-n)^{(n-1)} .
$$

However, unlike the case where the $G_{i}$ are finite, $\mathrm{WH}\left(F_{n}\right)$ is of infinite index in $\operatorname{AUT}\left(F_{n}\right)$. In fact, the cohomological dimension of $\mathrm{WH}\left(F_{n}\right)$ is $n-1$ [089], precisely half the vcd of $\operatorname{Aut}\left(F_{n}\right)$ CV86.

\section{Palindromic Automorphisms}

The palindromic automorphism group $\Pi A\left(F_{n}\right)$ of a free group $F_{n}=F\left(a_{1}, \ldots, a_{n}\right)$ was introduced by Collins Co95. A palindrome is a word $w_{1} w_{2} \ldots w_{n}$ in $F_{n}$ that is the same as its reverse $w_{n} \ldots w_{2} w_{1}$, and the group $\Pi A\left(F_{n}\right)$ consists of all automorphisms of $F_{n}$ that send each generator of $F_{n}$ to a palindrome. Collins derived a presentation for $\Pi A\left(F_{n}\right)$ using the following generators:

- Palindromic Whitehead moves $\left(a_{i} \| a_{j}\right), i \neq j$, which send $a_{i} \mapsto a_{j} a_{i} a_{j}$ and fix all other generators $a_{k}$.

- Factor automorphisms $\sigma_{a_{i}}$ which send $a_{i} \mapsto a_{i}^{-1}$ and fix all other generators $a_{k}$.

- Permutation automorphisms corresponding to elements of the symmetric group $\Sigma_{n}$ which permute the $a_{1}, \ldots, a_{n}$ among themselves.

The subgroup of $\Pi A\left(F_{n}\right)$ generated just by the palindromic Whitehead moves $\left(a_{i} \| a_{j}\right)$ is the elementary palindromic automorphism group $E \Pi A\left(F_{n}\right)$, and the subgroup generated by the palindromic Whitehead moves and the factor automorphisms is the pure palindromic automorphism group $P \Pi A\left(F_{n}\right)$.

In GJ00 Glover and Jensen observe that $\Pi A\left(F_{n}\right)$ is a centralizer of an involution: $\Pi A\left(F_{n}\right)=C_{\mathrm{AUT}\left(F_{n}\right)}\left(\sigma_{n}\right)$ where $\sigma_{n}$ is the automorphism that sends each $a_{i}$ to $a_{i}^{-1}$. This allows them to show that $E \Pi A\left(F_{n}\right)$ is torsion free, $\operatorname{cd}\left(E \Pi A\left(F_{n}\right)\right)=n-1$, as well as to find information on the cohomology of $\Pi A\left(F_{n}\right)$.

In this section we calculate the Euler characteristic of $\Pi A\left(F_{n}\right)$. We thank Andy Miller for first observing the following useful description of $\Pi A\left(F_{n}\right)$. Let $G=$ $*_{i=0}^{n} G_{i}$, where each $G_{i}=\left\langle x_{i}\right\rangle \cong \mathbb{Z}_{2}$. Denote by $\operatorname{AUT}\left(*_{i=0}^{n} \mathbb{Z}_{2}, x_{0}\right)$ the subgroup of $\operatorname{AUT}\left(*_{i=0}^{n} \mathbb{Z}_{2}\right)$ consisting of automorphisms $\phi \in \operatorname{AUT}\left(*_{i=0}^{n} \mathbb{Z}_{2}\right)$ that fix the first factor, $\phi\left(x_{0}\right)=x_{0}$.

Lemma 6.1. There is an isomorphism $\iota: \operatorname{AUT}\left(*_{i=0}^{n} \mathbb{Z}_{2}, x_{0}\right) \rightarrow \Pi A\left(F_{n}\right)$ satisfying $\iota\left(\operatorname{FR}\left(*_{i=0}^{n} \mathbb{Z}_{2}, x_{0}\right)\right)=P \Pi A\left(F_{n}\right)$.

Proof. For each $i=1, \ldots, n$, let $a_{i}=x_{0} x_{i}$ and let $F_{n}$ be the subgroup of $G$ generated by $a_{1}, \ldots, a_{n}$. Because $F_{n}$ is the subgroup of $G$ consisting of words of even length (in the $x_{i}$ ), it is a normal subgroup of $G$ and we have a short exact sequence

$$
1 \rightarrow F_{n} \rightarrow G \rightarrow \mathbb{Z}_{2} \rightarrow 1
$$


with a splitting given by sending $\mathbb{Z}_{2}$ to $G_{0}$. In fact, the subgroup of words of even length is necessarily a characteristic subgroup of $G$ and it is a free group on the $n$ generators $a_{1}, \ldots, a_{n}$.

Since $F_{n}$ is a characteristic subgroup, there is a map

$$
\iota: \operatorname{Aut}\left(G, x_{0}\right) \rightarrow \operatorname{Aut}\left(F_{n}\right) .
$$

Let $\phi \in \operatorname{Ker}(\iota)$. Then $\phi\left(a_{i}\right)=a_{i}$ for all $i=1, \ldots, n$ and $\phi\left(x_{0}\right)=x_{0}$. Hence for each index $i, \phi\left(x_{i}\right)=\phi\left(x_{0} x_{0} x_{i}\right)=\phi\left(x_{0}\right) \phi\left(x_{0} x_{i}\right)=x_{0} \phi\left(a_{i}\right)=x_{0} a_{i}=x_{0} x_{0} x_{i}=x_{i}$. Thus $\phi=1$, and we have shown that $\iota$ is injective.

Let $\sigma_{n} \in \operatorname{AUT}\left(F_{n}\right)$ be the automorphism that sends each $a_{i}$ to $a_{i}^{-1}$. We show that for each $\phi \in \operatorname{AUT}\left(G, x_{0}\right), \iota\left(\phi^{-1}\right) \sigma_{n} \iota(\phi)=\sigma_{n}$, so that $\iota(\phi) \in \Pi A\left(F_{n}\right)=$ $C_{\mathrm{AUT}\left(F_{n}\right)}\left(\sigma_{n}\right)$. By the Kuroš Subgroup Theorem, there exists a sequence $x_{i_{1}}, \ldots, x_{i_{m}}$ such that $\phi\left(x_{i}\right)=x_{i_{1}} \cdots x_{i_{m-1}} x_{i_{m}} x_{i_{m-1}} \cdots x_{i_{1}}$. Recall that we have defined $a_{i}=$ $x_{0} x_{i}$, and abusing notation, we set $a_{0}=x_{0} x_{0}=1 \in F_{n}$. Note that

$$
\begin{aligned}
{\left[\iota\left(\phi^{-1}\right) \sigma_{n} \iota(\phi)\right]\left(a_{i}\right) } & =\left[\iota\left(\phi^{-1}\right) \sigma_{n} \iota(\phi)\right]\left(x_{0} x_{i}\right) \\
& =\left[\iota\left(\phi^{-1}\right) \sigma_{n}\right]\left(x_{0} \phi\left(x_{i}\right)\right) \\
& =\left[\iota\left(\phi^{-1}\right) \sigma_{n}\right]\left(x_{0} x_{i_{1}} \cdots x_{i_{m-1}} x_{i_{m}} x_{i_{m-1}} \cdots x_{i_{1}}\right) \\
& =\left[\iota\left(\phi^{-1}\right) \sigma_{n}\right]\left(a_{i_{1}} a_{i_{2}}^{-1} a_{i_{3}} \cdots a_{i_{m-1}}^{ \pm 1} a_{i_{m}}^{\mp 1} a_{i_{m-1}}^{ \pm 1} \cdots a_{i_{1}}\right) \\
& =\iota\left(\phi^{-1}\right)\left(a_{i_{1}}^{-1} a_{i_{2}} a_{i_{3}}^{-1} \cdots a_{i_{m-1}}^{\mp 1} a_{i_{m}}^{ \pm 1} a_{i_{m-1}}^{\mp 1} \cdots a_{i_{1}}^{-1}\right) \\
& =\iota\left(\phi^{-1}\right)\left(x_{i_{1}} \cdots x_{i_{m-1}} x_{i_{m}} x_{i_{m-1}} \cdots x_{i_{1}} x_{0}\right) \\
& =x_{i} x_{0} \\
& =a_{i}^{-1},
\end{aligned}
$$

so the injection $\iota$ restricts to

$$
\iota: \operatorname{Aut}\left(G, x_{0}\right) \rightarrow \Pi A\left(F_{n}\right) .
$$

We show that $\iota$ is a surjection by showing that the generators of $\Pi A\left(F_{n}\right)$ are in the image of $\iota$. For each pair $i, j$ of distinct integers from $1, \ldots, n$, let $\phi_{i, j} \in$ $\operatorname{Aut}\left(G, x_{0}\right)$ be the automorphism sending $x_{k}$ to $x_{0} x_{k} x_{0}$ for $k \neq i, 0$ and $x_{i}$ to $x_{j} x_{0} x_{i} x_{0} x_{j}$. Then $\iota\left(\phi_{i, j}\right)=\left(a_{i} \| a_{j}\right)$. The permutation automorphisms of $\Pi A\left(F_{n}\right)$ can be realized as maps fixing $x_{0}$ and permuting the $x_{0} x_{i} x_{0}$. Finally, the factor automorphism that inverts $x_{0} x_{i}$ and fixes all other generators corresponds to the map where $x_{i} \mapsto x_{0} x_{i} x_{0}$ and all other generators are fixed. So $\iota$ is an isomorphism.

The above calculations show that $\iota$ sends $\operatorname{FR}\left(*_{i=0}^{k} G_{i}, x_{0}\right)$ to the pure palindromic automorphism group $P \Pi A\left(F_{n}\right)$ generated by palindromic Whitehead moves and factor automorphisms.

Theorem C. If $F_{n}$ is a free group of rank $n$, then the Euler characteristics of the elementary palindromic automorphism group of $F_{n}$, the pure palindromic automorphism group of $F_{n}$, and the palindromic automorphism group of $F_{n}$ are as follows:

$$
\begin{aligned}
\chi\left(E \Pi A\left(F_{n}\right)\right) & =(1-n)^{n-1}, \\
\chi\left(P \Pi A\left(F_{n}\right)\right) & =\frac{(1-n)^{n-1}}{2^{n}}, \\
\chi\left(\Pi A\left(F_{n}\right)\right) & =\frac{(1-n)^{n-1}}{2^{n} \cdot n !} .
\end{aligned}
$$


Proof. Let $G$ denote the free product of $n+1$ copies of $\mathbb{Z}_{2}$ and suppose the first $\mathbb{Z}_{2}$ is generated by $x_{0}$. Conjugation by $x_{0}$ is the only inner automorphism in $P \Pi A\left(F_{n}\right)=\operatorname{FR}\left(G, x_{0}\right)=\mathrm{WH}\left(G, x_{0}\right)$, and we have a short exact sequence

$$
1 \rightarrow \mathbb{Z}_{2} \rightarrow P \Pi A\left(F_{n}\right) \rightarrow \mathrm{OWH}\left(G, x_{0}\right) \rightarrow 1 .
$$

Clearly $\operatorname{OWh}\left(G, x_{0}\right)<\mathrm{OWh}(G)$. However, we can conjugate each generator of $\mathrm{WH}(G)$ to form a generating set for $\mathrm{OWH}(G)$, each element of which fixes $x_{0}$. Hence $\mathrm{OWH}\left(G, x_{0}\right)=\mathrm{OWH}(G)$, and therefore the Euler characteristic of $P \Pi A\left(F_{n}\right)$ is

$$
\chi\left(P \Pi A\left(F_{n}\right)\right)=\chi\left(\mathbb{Z}_{2}\right) \cdot \chi(\mathrm{OWH}(G))=(1 / 2) \cdot((1-n) / 2)^{n-1}=\frac{(1-n)^{n-1}}{2^{n}} .
$$

The remaining assertions follow from the short exact sequences

$$
1 \rightarrow E \Pi A\left(F_{n}\right) \rightarrow P \Pi A\left(F_{n}\right) \rightarrow\left(\mathbb{Z}_{2}\right)^{n} \rightarrow 1
$$

and

$$
1 \rightarrow P \Pi A\left(F_{n}\right) \rightarrow \Pi A\left(F_{n}\right) \rightarrow \Sigma_{n} \rightarrow 1
$$

and the fact that $\chi\left(\left(\mathbb{Z}_{2}\right)^{n}\right)=\left(\frac{1}{2}\right)^{n}$ and $\chi\left(\Sigma_{n}\right)=\frac{1}{n !}$.

Remark 6.2. The groups $\mathrm{P} \Sigma_{n}$ and $E \Pi A\left(F_{n}\right)$ are both torsion free groups of cohomological dimension $n-1$, and they have very similar presentations. By Theorem $\mathrm{C}$ they also have the same Euler characteristic. This might lead an optimist to think these groups are isomorphic, or perhaps a more meek conjecture would be that they have isomorphic homology. However, as was noted in GJ00, $\mathrm{P} \Sigma_{n}$ abelianizes to a free abelian group of rank $n(n-1)$ while $E \Pi A\left(F_{n}\right)$ abelianizes to an elementary abelian 2-group of rank $n(n-1)$.

Borrowing an argument of Smillie and Vogtmann - used to establish Proposition 6.4 in [SV87] - we can show that the kernel of the linearization map is not of finite homological type. As was first pointed out in Co95], the image of $P \Pi A\left(F_{n}\right)$ in the general linear group is the congruence subgroup $\widetilde{\Gamma}_{2}(\mathbb{Z})$ defined by the short exact sequence

$$
1 \rightarrow \widetilde{\Gamma}_{2}(\mathbb{Z}) \rightarrow G L_{n}(\mathbb{Z}) \rightarrow G L_{n}\left(\mathbb{Z}_{2}\right) \rightarrow 1 .
$$

Thus if the kernel $K$ of $P \Pi A\left(F_{n}\right) \rightarrow \widetilde{\Gamma}_{2}(\mathbb{Z})$ were of finite homological type, then by item 2 of Theorem 4.1 we would have

$$
\chi\left(P \Pi A\left(F_{n}\right)\right)=\chi(K) \cdot \chi\left(\widetilde{\Gamma}_{2}(\mathbb{Z})\right)=0
$$

since $\chi\left(\tilde{\Gamma}_{2}(\mathbb{Z})\right)=0$ by Harder Ha71]. But this contradicts Theorem $\mathbb{C}$ so the kernel cannot have finite homological type.

Corollary 6.3. For $n \geq 3$, the kernel of the map from $P \Pi A\left(F_{n}\right)$ to $G L_{n}(\mathbb{Z})$ does not have finitely generated integral homology.

As in the case of the famous kernel $\mathrm{IA}_{n}=\operatorname{ker}\left[\operatorname{AUt}\left(F_{n}\right) \rightarrow G L_{n}(\mathbb{Z})\right]$, it would be interesting to know what finiteness properties these kernels do enjoy.

\section{REFERENCES}

[Br74] Kenneth S. Brown. Euler characteristics of discrete groups and G-spaces. Invent. Math., 27:229-264, 1974. MR0385007 (52:5877)

[Br94] Kenneth S. Brown. Cohomology of groups, volume 87 of Graduate Texts in Mathematics. Springer-Verlag, New York, 1994. Corrected reprint of the 1982 original. MR1324339 (96a:20072) 


$$
\chi\left(\mathrm{WH}\left(G_{1} * \cdots * G_{n}\right)\right)=\chi\left(G_{1} * \cdots * G_{n}\right)^{n-1}
$$

[Co89] Donald J. Collins. Cohomological dimension and symmetric automorphisms of a free group. Comment. Math. Helv., 64(1):44-61, 1989. MR0982561 (90e:20035)

[Co95] Donald J. Collins. Palindromic automorphisms of free groups. In Combinatorial and geometric group theory (Edinburgh, 1993), volume 204 of London Math. Soc. Lecture Note Ser., pages 63-72. Cambridge Univ. Press, Cambridge, 1995. MR.1320275 (96c:20048)

[CL83] Donald J. Collins and Frank Levin. Automorphisms and Hopficity of certain BaumslagSolitar groups. Arch. Math. (Basel), 40(5):385-400, 1983. MR0707725 (85b:20043)

[CV86] Marc Culler and Karen Vogtmann. Moduli of graphs and automorphisms of free groups. Invent. Math., 84(1):91-119, 1986. MR0830040 (87f:20048)

[GJ00] Henry H. Glover and Craig A. Jensen. Geometry for palindromic automorphism groups of free groups. Comment. Math. Helv., 75(4):644-667, 2000. MR1789180(2002m:20058)

[GKP94] Ronald L. Graham, Donald E. Knuth, and Oren Patashnik. Concrete mathematics. Addison-Wesley Publishing Company, Reading, MA, second edition, 1994. A foundation for computer science. MR.1397498 (97d:68003)

[Ha71] G. Harder. A Gauss-Bonnet formula for discrete arithmetically defined groups. Ann. Sci. École Norm. Sup. (4), 4:409-455, 1971. MR0309145 (46:8255)

[HZ86] J. Harer and D. Zagier. The Euler characteristic of the moduli space of curves. Invent. Math., 85(3):457-485, 1986. MR0848681 (87i:32031)

[KV93] Sava Krstić and Karen Vogtmann. Equivariant outer space and automorphisms of freeby-finite groups. Comment. Math. Helv., 68(2):216-262, 1993. MR.1214230 (94c:20067)

[MM04] Jon McCammond and John Meier. The hypertree poset and the $l^{2}$-Betti numbers of the motion group of the trivial link. Math. Ann., 328(4):633-652, 2004. MR2047644 (2005b:20104)

[MM96] Darryl McCullough and Andy Miller. Symmetric automorphisms of free products. Mem. Amer. Math. Soc., 122(582):viii+97, 1996. MR1329943 (96k:20069)

[Pe88] R. C. Penner. Perturbative series and the moduli space of Riemann surfaces. J. Differential Geom., 27(1):35-53, 1988. MR0918455 (89h:32045)

[Ro84] Steven Roman. The umbral calculus, volume 111 of Pure and Applied Mathematics. Academic Press Inc. [Harcourt Brace Jovanovich Publishers], New York, 1984. MR0741185 (87c:05015)

[Se79] J.-P. Serre. Arithmetic groups. In Homological group theory (Proc. Sympos., Durham, 1977), volume 36 of London Math. Soc. Lecture Note Ser., pages 105-136. Cambridge Univ. Press, Cambridge, 1979. MR0564421 (82b:22021)

[SV87] John Smillie and Karen Vogtmann. A generating function for the Euler characteristic of Out $\left(F_{n}\right)$. J. Pure Appl. Algebra, 44(1-3):329-348, 1987. MR0885116 (88g:20107)

[St99] Richard P. Stanley. Enumerative combinatorics. Vol. 2, volume 62 of Cambridge Studies in Advanced Mathematics. Cambridge University Press, Cambridge, 1999. With a foreword by Gian-Carlo Rota and appendix 1 by Sergey Fomin. MR:1676282 (2000k:05026)

Department of Mathematics, University of New Orleans, New Orleans, Louisiana 70148

E-mail address: jensen@math.uno.edu

Department of Mathematics, University of California, Santa Barbara, California 93106

E-mail address: jon.mccammond@math.ucsb.edu

Department of Mathematics, Lafayette College, Easton, Pennsylvania 18042

E-mail address: meierj@lafayette.edu 